\title{
Deutscher Bibliotheksverband begrüßt die Botschaft der Bundeskanzlerin zur Bedeutung des Lesens
}

http://doi.org/10.1515/bd-2018-0050

Kurz vor Eröffnung der Leipziger Buchmesse hat Bundeskanzlerin Angela Merkel die Bedeutung des Lesens unterstrichen. „Auch in der Zeit der Digitalisierung ist das Lesen eine Grundkompetenz, die einem Vieles im Leben erschließt“, sagt Merkel in ihrem neuen Video-Podcast. „Deshalb müssen wir auch im 21. Jahrhundert auf das Lesen viel Wert legen.“

Angesichts von 7,5 Mio. funktionalen Analphabeten in Deutschland ist die verstärkte Förderung des Bundes für die Schulbildung eine begrüßenswerte und dringend notwendige Unterstützung. Dabei leisten Bibliotheken als herausragende Orte der außerschulischen Leseförderung und als Kultur- und Bildungspartner von Kindertagesstätten, Schulen und weiteren Einrichtungen flächendeckend einen wichtigen Beitrag. Der breite Medienbestand in Bibliotheken bietet für Familien, Erzieher/innen und Lehrer/innen eine große Auswahl an Lesestoff und Lernmaterialien in allen Formaten. Zahlreiche Leseveranstaltungen, auch mit digitalen Medien, werden täglich von Kinder- und Jugendbibliothekar/innen, ehrenamtlichen Vorlesepatinnen und -paten oder Autor/innen in unseren Bibliotheken durchgeführt. Angesichts abnehmender Lesekompetenz ist eine frühe und abgestimmte Förderung durch alle relevanten Einrichtungen unabdingbar. Bibliotheken leisten täglich einen unaufgeregten, aber höchst wirkungsvollen Beitrag, damit Deutschland als Bildungsnation gestärkt wird.

„Wir begrüßen daher ausdrücklich die Ankündigung der künftigen Bundesregierung im Koalitionsvertrag zu prüfen, wie der Bund zum Erhalt der vielfältigen Bibliothekslandschaft und ihrer zunehmend gesellschaftlichen Bedeutung beitragen kann,“ so die Bundesvorsitzende des Deutschen Bibliotheksverbandes Barbara Lison. „Bibliotheken sollten auch im digitalen Zeitalter ihre zentralen Funktionen für Bildung und Kultur erfüllen können“, wird im Koalitionsvertrag unterstrichen.

Barbara Lison fordert daher: „Um die Lesefähigkeit aller Generationen besser zu fördern, muss das Potential der Bibliotheken voll ausgeschöpft werden. Die Kooperation zwischen Schulen, Kitas und Bibliotheken muss weiter ausgebaut und die notwendigen Ressourcen müssen erweitert werden. Ziel muss sein, diese Kooperationen in die Lehr- und Bildungsrahmenpläne jedes Bundeslandes als verbindliche Maßnahme für alle Klassenstufen einzubringen. Unter diesen 
Bedingungen kann die Zusammenarbeit vereinfacht und verstetigt werden, so dass möglichst alle Kinder und Jugendliche in jeder Kommune von den Bibliotheksangeboten profitieren können.“

Der Video-Podcast ist unter www.bundeskanzlerin.de abrufbar.

Kontakt: Deutscher Bibliotheksverband e. V.

Maiken Hagemeister, Pressesprecherin und Leitung Kommunikation, Tel.: 0 30/6449899 25

E-Mail: hagemeister@bibliotheksverband.de,www.bibliotheksverband.de, www.bibliotheksportal.de 